\title{
UPPER BOUND ON THE PROTON LIFETIME AND THE MINIMAL NON-SUSY GRAND UNIFIED THEORY*
}

\author{
Pavel Fileviez Péręt \\ Centro de Física Teórica de Partículas. Departamento de \\ Física. Instituto Superior Técnico. Avenida Rovisco Pais, 1 \\ 1049-001 Lisboa, Portugat
}

\begin{abstract}
In this talk we show that it is possible to find an upper bound on the total proton lifetime. We conclude that the minimal realistic non-supersymmetric grand unified theory is the modified Georgi-Glashow model with a Higgs sector composed of $5_{H}, 24_{H}$, and $15_{H}$. We discuss the possibility to test this scenario at the next generation of proton decay experiments and future colliders through the production of light scalar leptoquarks.
\end{abstract}

\section{INTRODUCTION}

It is well-known that the decay of the proton is the most dramatic prediction coming from matter unification [1]. For this reason we believe that the best way to test the grand unified theories is through proton decay.

In the first part of my talk I will discuss the possibility to find an upper bound on the proton lifetime, which is crucial to understand the possibility to test the different minimal grand unified models with the standard model matter content.

As we know the minimal Georgi-Glashow model 2] is the simplest grand unified theory. However, this model is not realistic. In the second part of my talk I will present the minimal realistic extension of the Georgi-Glashow theory. I will show that this model could be tested at future proton decay experiments since the upper bound on the proton decay lifetime is $\tau_{p} \leq 1.4 \times 10^{36}$ years. This model predicts light scalar leptoquarks, therefore for the first time we have the possibility to test a GUT model at future hadron colliders, particularly at the LHC.

\footnotetext{
* Based on the Talks given at NURT'06 (V International Symposium on Nuclear and Related Techniques, Havana, CUBA, 3-7 April 2006), PLANCK'06 (The ninth European meeting "From the Planck scale to the ElectroWeak scale", Paris, FRANCE, 29 May 29 - 2 June,2006) and SUSY'06 (14th International Conference on Supersymmetry and the Unification of Fundamental Interactions. Irvine, California, USA. 12-17 June 2006).

${ }^{\dagger}$ Electronic address: fileviez@cftp.ist.utl.pt

${ }^{\ddagger} \mathrm{URL}:$ http://cftp.ist.utl.pt/ fileviez
} 


\section{UPPER BOUND ON THE TOTAL PROTON LIFETIME}

There are several relevant operators to the decay of the proton. In non-supersymmetric grand unified theories we have the gauge and Higgs $d=6$ contributions. It is well-known that the gauge contributions are the most important in this context. In supersymmetric models the most important contributions are coming from the $d=4$ and $d=5$ operators (For details see [3]). As we know one can always forbid or suppress the $d=4, d=5$, and the Higgs $d=6$ contributions. However, it is not possible to forbid the gauge contributions in grand unified models with the standard model matter content. Now, since those contributions are always present and are the least model dependent, the upper bound on the proton lifetime is coming from the minimization of the decay rate due to the presence of those operators.

In order to minimize the decay rate due to the presence of gauge $d=6$ contributions let us study those in details. In the physical basis these operators read as [4]:

$$
\begin{aligned}
O\left(e_{\alpha}^{C}, d_{\beta}\right) & =c\left(e_{\alpha}^{C}, d_{\beta}\right) \epsilon_{i j k} \overline{u_{i}^{C}} \gamma^{\mu} u_{j} \overline{e_{\alpha}^{C}} \gamma_{\mu} d_{k \beta}, \\
O\left(e_{\alpha}, d_{\beta}^{C}\right) & =c\left(e_{\alpha}, d_{\beta}^{C}\right) \epsilon_{i j k} \overline{u_{i}^{C}} \gamma^{\mu} u_{j} \overline{d_{k \beta}^{C}} \gamma_{\mu} e_{\alpha}, \\
O\left(\nu_{l}, d_{\alpha}, d_{\beta}^{C}\right) & =c\left(\nu_{l}, d_{\alpha}, d_{\beta}^{C}\right) \epsilon_{i j k} \overline{u_{i}^{C}} \gamma^{\mu} d_{j \alpha} \overline{d_{k \beta}^{C}} \gamma_{\mu} \nu_{l}
\end{aligned}
$$

where

$$
\begin{aligned}
c\left(e_{\alpha}^{C}, d_{\beta}\right) & =k_{1}^{2}\left[V_{1}^{11} V_{2}^{\alpha \beta}+\left(V_{1} V_{U D}\right)^{1 \beta}\left(V_{2} V_{U D}^{\dagger}\right)^{\alpha 1}\right] \\
c\left(e_{\alpha}, d_{\beta}^{C}\right) & =k_{1}^{2} V_{1}^{11} V_{3}^{\beta \alpha}+k_{2}^{2}\left(V_{4} V_{U D}^{\dagger}\right)^{\beta 1}\left(V_{1} V_{U D} V_{4}^{\dagger} V_{3}\right)^{1 \alpha} \\
c\left(\nu_{l}, d_{\alpha}, d_{\beta}^{C}\right) & =k_{1}^{2}\left(V_{1} V_{U D}\right)^{1 \alpha}\left(V_{3} V_{E N}\right)^{\beta l}+k_{2}^{2} V_{4}^{\beta \alpha}\left(V_{1} V_{U D} V_{4}^{\dagger} V_{3} V_{E N}\right)^{1 l}
\end{aligned}
$$

$\alpha=\beta=1,2 ; l=1,2,3$, and $k_{1,2}=g_{G U T} / \sqrt{2} M_{V, V^{\prime}}$. In our notation $g_{G U T}$ is the gauge coupling at the GUT scale, and $M_{V, V^{\prime}}$ the mass of the gauge bosons $(X, Y)=(\mathbf{3}, \mathbf{2}, 5 / 3),\left(X^{\prime}, Y^{\prime}\right)=$ $(\mathbf{3}, \mathbf{2},-1 / 3)$, respectively. The relevant mixing matrices for the above equations are defined by $V_{1}=U_{C}^{\dagger} U, V_{2}=E_{C}^{\dagger} D, V_{3}=D_{C}^{\dagger} E, V_{4}=D_{C}^{\dagger} D, V_{E N}=E^{\dagger} N=K_{3} V_{P M N S}$, and $V_{U D}=U^{\dagger} D=$ $K_{1} V_{C K M} K_{2}$, once we define the diagonalization of the Yukawa matrices as: $U_{C}^{T} Y_{U} U=Y_{U}^{d i a g}$, $D_{C}^{T} Y_{D} D=Y_{D}^{d i a g}$, and $E_{C}^{T} Y_{E} E=Y_{E}^{d i a g}$. Here it is important to understand that it is difficult to predict the lifetime of the proton since we do not know all mixing matrices mentioned above. Even in minimal models it is very difficult to predict all those matrices.

Now, we would like to find the minimum of the decay rate. Let us study the case of grand unified theories based on $S U(5)$ gauge symmetry $\left(k_{2}=0\right)$. In order to find a possible "minimum" 
of the decay rate in these theories, we study two major cases [5]. In the first case there are no decays into a meson and antineutrinos, and in the second there are no decays into a meson and charged antileptons. We investigated those cases in detail, showing that the upper bound is coming from the first case [5]. Let us understand these results. In order to set to zero all channels with antineutrinos we have to look for a model of fermion masses where the following conditions are valid [5]:

$$
\begin{aligned}
\left(V_{1} V_{U D}\right)^{1 \alpha} & =\left(U_{C}^{\dagger} D\right)^{1 \alpha}=0 \Longrightarrow U_{C}=D A^{\dagger} \quad(\mathrm{C} . \mathrm{I}) \\
V_{2}^{\beta \alpha} & =\left(E_{C}^{\dagger} D\right)^{\beta \alpha}=0 \Longrightarrow E_{C}=D B_{1} \quad(\mathrm{C} . \mathrm{II}) \\
V_{3}^{\beta \alpha} & =\left(D_{C}^{\dagger} E\right)^{\beta \alpha}=0 \Longrightarrow D_{C}=E B_{2} \quad(\mathrm{C} . \mathrm{III})
\end{aligned}
$$

with

$$
A=\left(\begin{array}{ccc}
0 & 0 & e^{i \alpha} \\
\ldots & \ldots & 0 \\
\ldots & \ldots & 0
\end{array}\right), B_{i}=\left(\begin{array}{ccc}
0 & 0 & e^{i \beta_{i}} \\
0 & e^{i \gamma_{i}} & 0 \\
e^{i \delta_{i}} & 0 & 0
\end{array}\right)
$$

In this case the decay rate is given by:

$$
\Gamma_{p}=\Gamma\left(p \rightarrow K^{0} \mu^{+}\right)=8 \pi^{2} C\left(p, K^{0}\right)\left|V_{C K M}^{13}\right|^{2} \alpha_{G U T}^{2} M_{V}^{-4}
$$

where

$$
C\left(p, K^{0}\right)=\frac{\left(m_{p}^{2}-m_{K}^{2}\right)^{2}}{8 \pi m_{p}^{3} f_{\pi}^{2}} A_{L}^{2}|\beta|^{2} \times\left[1+\frac{m_{p}}{m_{B}}(D-F)\right]^{2}
$$

See reference [5] for the notation. Therefore the upper bound on the proton lifetime reads as:

$$
\tau_{p} \leq 6.0 \times 10^{39} \frac{\left(M_{V} / 10^{16} \mathrm{GeV}\right)^{4}}{\alpha_{G U T}^{2}}\left(0.003 \mathrm{GeV}^{3} / \beta\right)^{2} \text { years }
$$

Notice that this expression is valid for a given value of the GUT scale and $\alpha_{G U T}$. Now, using the experimental lower bound $\left(\tau_{p} \geq 50 \times 10^{32}\right.$ years $)$ and the value $\beta=0.003 \mathrm{GeV}^{3}$ for the matrix element, we can conclude that the lower bound on the GUT scale is $M_{V}>3.04 \times 10^{14} \sqrt{\alpha_{G U T}} \mathrm{GeV}$. Now, if we use the values for $\alpha_{G U T}=1 / 39-1 / 25$ in the non-susy and susy case, respectively:

$$
M_{V}>(4.9-6.1) \times 10^{13} \mathrm{GeV}
$$

Notice that the lower bound on the GUT scale tells us that a non-SUSY $S U(5)$ could be consistent with the experimental bounds. 
In flipped $S U(5)$ theories [6] $\left(k_{1}=0\right)$ it is possible to set to zero all proton decay channels at the same time [7]. Therefore, the above upper bound on the proton lifetime is also valid for $S O(10)$ theories.

Now, we can make a plot (Figure 1) to understand when a given grand unified theory is ruled out by the experimental lower bounds on the proton lifetime.

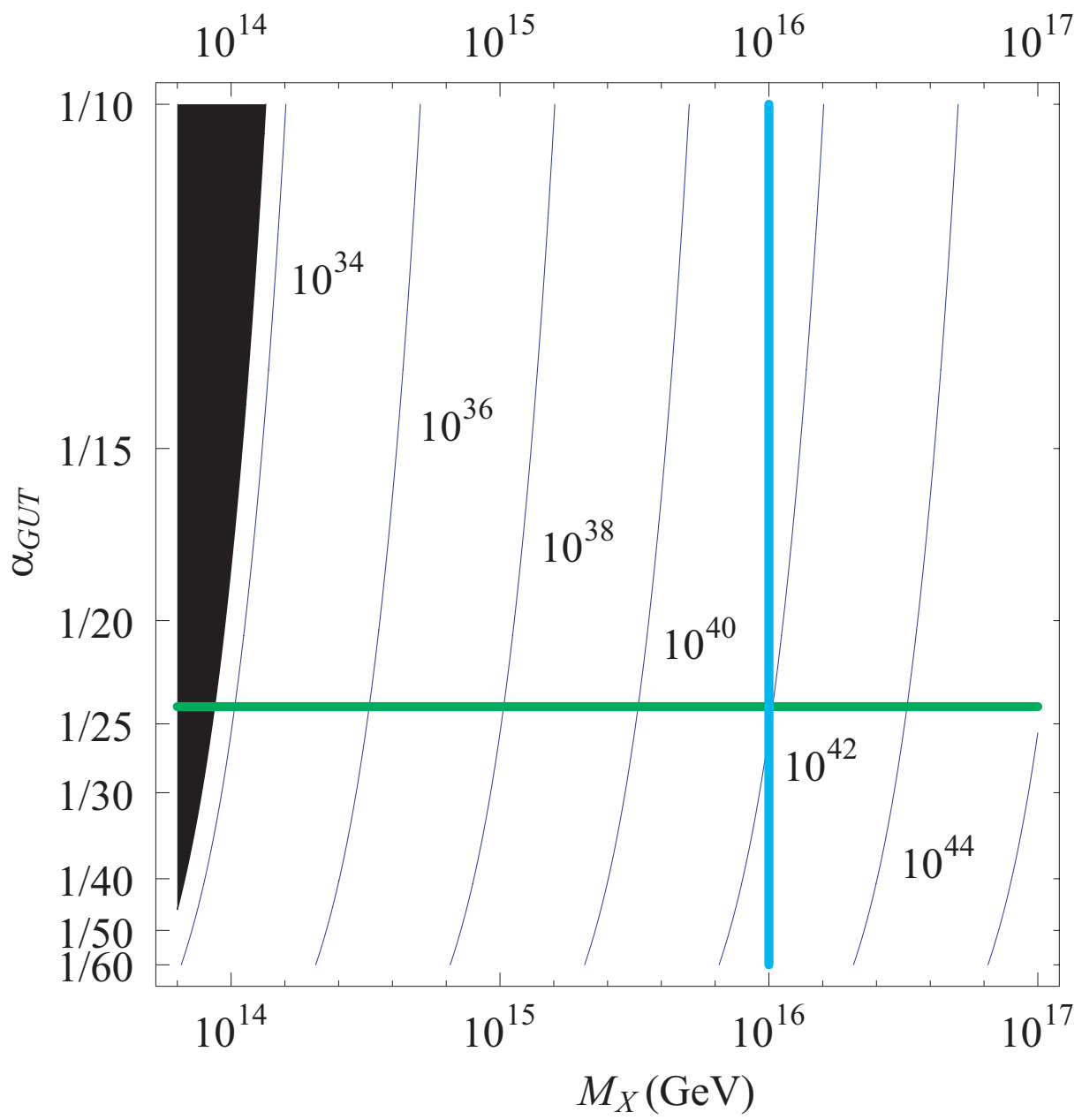

FIG. 1: Isoplot for the upper bounds on the total proton lifetime in years in the Majorana neutrino case in the $M_{X}-\alpha_{G U T}$ plane. The value of the unifying coupling constant is varied from 0.02 to 1 [5].

Notice that from Figure 1 we can conclude that the non-supersymmetric grand unified theories are still in agreement with the proton decay experiments. Now, since in future experiments the bounds on the proton decay experiments could be improved in two orders of magnitude, we are not sure about the possibility to test the supersymmetric grand unified theories through nucleon decay where the $d=5$ operators are absent or suppressed. In other words, in this case the lifetime of the proton could be very large. 
As we discussed before the non-susy theories could be realistic. Then, let us study the possibility to write down the simplest non-susy grand unified theory.

\section{THE MINIMAL REALISTIC EXTENSION OF THE GEORGI-GLASHOW MODEL}

In the Georgi-Glashow model based on $S U(5)$ gauge symmetry [2] the standard model matter is unified in the $\overline{5}$ and 10 representations and the Higgs sector is composed of the $5_{H}$ and $24_{H}$. As it is well-known this model is not realistic since it is not possible to unify all gauge couplings, the neutrinos are massless, and we find the wrong relation $Y_{D}=Y_{E}^{T}$. Now, as we pointed out in reference 8 ], if we add the $15_{H}$ and use the higher-dimensional operators, we can write the minimal realistic extension of the Georgi-Glashow model, where it is possible to achieve unification, we can use the type II see-saw mechanism [9] for neutrino masses and it is possible to find a consistent relation between the fermion masses in this context.

We studied the unification constraints in detail [10]. In Figure 2 we show the whole parameter space where it is possible to achieve unification in agreement with all low energy data.

Notice the different values for the masses of the fields which help us to achieve unification. Those are $\Sigma_{3}=(1,3,0) \subset 24_{H}, \Phi_{a}=(1,3,1) \subset 15_{H}$, and $\Phi_{b}=(3,2,1 / 6) \subset 15_{H}$. In this model the maximal value for the unification scale is $M_{G U T}=3.2 \times 10^{14} \mathrm{GeV}$, defined for $M_{\Sigma_{8}}=10^{5} \mathrm{GeV}$, $M_{\Sigma_{3}}=M_{Z}, M_{\Phi_{a}}=130 \mathrm{GeV}, M_{\Phi_{b}}=242 \mathrm{GeV}$, and $\alpha_{G U T}=1 / 37.3$. See references [8] and [10] for details. Now, using the maximal value for the GUT scale the upper bound on the proton lifetime reads as:

$$
\tau_{p} \leq 1.4 \times 10^{36} \text { years }
$$

Therefore we can say that this model could be tested or ruled out at the next generation of proton decay experiments.

Recently, we studied the simplest renormalizable realistic $S U(5)$ model which Higgs sector is composed of $5_{H}, 24_{H}$, and $45_{H}$ [1] , and we concluded that the proton lifetime can be very large. Therefore, we can say that the only realistic non-SUSY SU(5) that we can verify in near future is the model presented in reference [8].

Now, if we study carefully the results presented in Figure 2, we see that once we impose the proton decay constraints we get an upper bound on the scalar leptoquark mass, which is basically $M_{\Phi_{b}} \leq 10^{8} \mathrm{GeV}$. Also notice that in the case of the most natural implementation of the type II seesaw mechanism (large $M_{\Phi_{a}}$ ) the mass of the scalar leptoquarks $\left(\Phi_{b}\right)$ is in the phenomenologically 


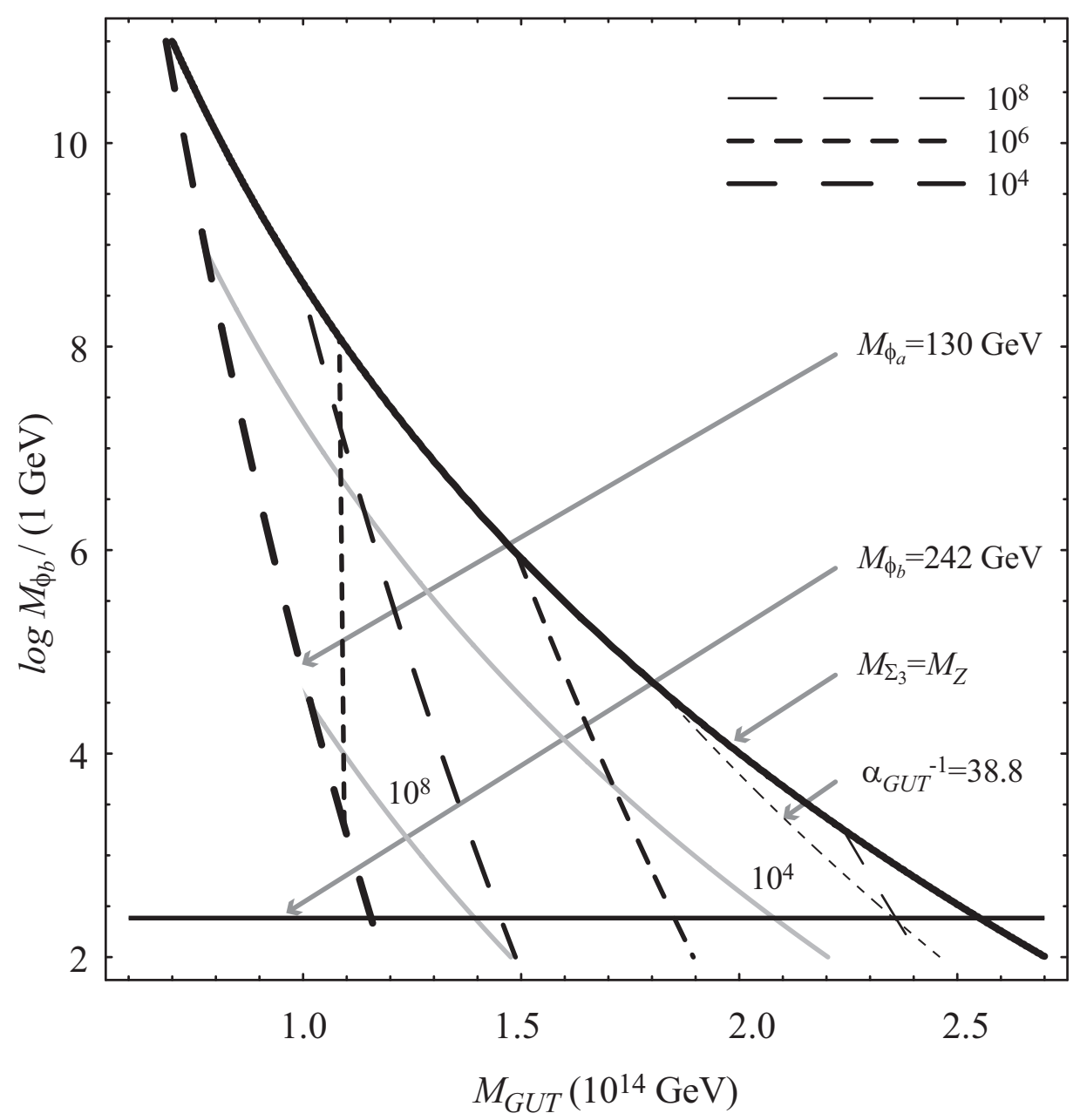

FIG. 2: Plot of lines of constant $M_{\Sigma_{3}}$ and $M_{\Phi_{a}}$ in the $M_{G U T}-\log \left(M_{\Phi_{b}} / 1 \mathrm{GeV}\right)$ plane, assuming exact oneloop unification. We use the central values for the gauge couplings as given in the text. All the masses are given in $\mathrm{GeV}$ units. The triangular region is bounded from the left (below) by the experimental limit on $M_{\Phi_{a}}\left(M_{\Phi_{b}}\right)$. The right bound is $M_{\Sigma_{3}} \geq M_{Z}$. The two grey solid (thick dashed) lines are the lines of constant $M_{\Sigma_{3}}\left(M_{\Phi_{a}}\right)$. The line of constant $\alpha_{G U T}^{-1}$ is also shown. The region to the left of the vertical dashed line is excluded by the proton decay experiments if $\beta=0.015 \mathrm{GeV}^{3}[10]$.

interesting region $\left(O\left(10^{2}-10^{3}\right) \mathrm{GeV}\right)$. Then, we can conclude that our scenario has a potential to be tested at the next generation of collider experiments, particularly at the Large Hadron Collider (LHC) at CERN. See reference [12] for the study of leptoquarks at the LHC.

Notice that usually it is very difficult to be sure about the possibility to test any grand unified theory through proton decay. However, here we have the exciting possibility to verify the idea of the unification of the gauge interactions at future colliders. 


\section{SUMMARY}

In this talk I have discussed the possibility to find an upper bound on the proton decay lifetime. The conservative upper bound on the total proton decay valid for any grand unified model with the standard model matter content is given by:

$$
\tau_{p} \leq 6.0 \times 10^{39} \frac{\left(M_{V} / 10^{16} \mathrm{GeV}\right)^{4}}{\alpha_{G U T}^{2}}\left(0.003 \mathrm{GeV}^{3} / \beta\right)^{2} \text { years }
$$

where $M_{V}$ is the mass of the superheavy gauge bosons and $\beta$ is the value of the matrix element.

We conclude that the non-supersymmetric grand unified theories with low unification scale $\left(M_{V}>4.9 \times 10^{13} \mathrm{GeV}\right)$ are not ruled out by the bounds coming from the proton decay searches.

It is shown that the minimal non-supersymmetric grand unified theory is the modified GeorgiGlashow model with Higgs sector composed of $5_{H}, 24_{H}$, and $15_{H}$. This scenario can be tested at future proton decay experiments $\left(\tau_{p} \leq 1.4 \times 10^{36}\right.$ years $)$ and future colliders, particularly at LHC, through the production of scalar leptoquarks $\Phi_{b}$.

\section{Acknowledgments}

I would like to thank the organizers of NURT'06 in Havana, PLANCK'06 in Paris, and SUSY'06 in Irvine for the possibility to present my work and for those stimulating conferences. This work has been supported by Fundação para a Ciência e a Tecnologia (FCT, Portugal) through the project CFTP, POCTI-SFA-2-777 and a fellowship under project POCTI/FNU/44409/2002. I would like to thank I. Dorsner and R. González Felipe for many discussions and enjoyable collaboration. I would like to thank B. Bajc, F. Feruglio, M. Frigerio, W. Hollik, S. Lavignac, P. Nath, M. Nebot, G. Rodrigo Garcia, C. Savoy, and G. Senjanović for discussions and comments.

[1] J. C. Pati and A. Salam, "Is Baryon Number Conserved?," Phys. Rev. Lett. 31 (1973) 661.

[2] H. Georgi and S. L. Glashow, "Unity Of All Elementary Particle Forces," Phys. Rev. Lett. 32 (1974) 438.

[3] P. Nath and P. Fileviez Pérez, "Proton stability in grand unified theories, in strings, and in branes," arXiv:hep-ph/0601023

[4] P. Fileviez Pérez, "Fermion mixings vs d $=6$ proton decay," Phys. Lett. B 595 (2004) 476 arXiv:hep-ph/0403286. 
[5] I. Dorsner and P. Fileviez Pérez, "How long could we live?," Phys. Lett. B 625 (2005) 88 arXiv:hep-ph/0410198.

[6] S. M. Barr, "A New Symmetry Breaking Pattern For SO(10) And Proton Decay," Phys. Lett. B 112 (1982) 219.

A. De Rujula, H. Georgi and S. L. Glashow, "Flavor Goniometry By Proton Decay," Phys. Rev. Lett. 45 (1980) 413.

[7] I. Dorsner and P. Fileviez Pérez, "Could we rotate proton decay away?," Phys. Lett. B 606 (2005) 367 arXiv:hep-ph/0409190.

[8] I. Dorsner and P. Fileviez Pérez, "Unification without supersymmetry: Neutrino mass, proton decay and light leptoquarks," Nucl. Phys. B 723 (2005) 53 arXiv:hep-ph/0504276.

[9] Type I seesaw: P. Minkowski, "Mu $\rightarrow$ E Gamma At A Rate Of One Out Of 1-Billion Muon Decays?," Phys. Lett. B 67 (1977) 421; T. Yanagida, proceedings of the Workshop on Unified Theories and Baryon Number in the Universe, Tsukuba, 1979, eds. A. Sawada, A. Sugamoto, KEK Report No. 79-18, Tsukuba. S. Glashow, in Quarks and Leptons, Cargèse 1979, eds. M. Lévy. et al., (Plenum, 1980, N ew York). M. Gell-Mann, P. Ramond, R. Slansky, proceedings of the Supergravity Stony Brook Workshop, New York, 1979, eds. P. Van Niewenhuizen, D. Freeman (North-Holland, Amsterdam). R. Mohapatra, G. Senjanović, "Neutrino Mass And Spontaneous Parity Nonconservation," Phys.Rev.Lett. 44 (1980) 912.

Type II seesaw: G. Lazarides, Q. Shafi and C. Wetterich, "Proton Lifetime And Fermion Masses In An SO(10) Model," Nucl. Phys. B 181 (1981) 287. R. N. Mohapatra and G. Senjanovic, "Neutrino Masses And Mixings In Gauge Models With Spontaneous Parity Violation," Phys. Rev. D 23 (1981) 165.

[10] I. Dorsner, P. Fileviez Pérez and R. González Felipe, "Phenomenological and cosmological aspects of a minimal GUT scenario," Nucl. Phys. B 747 (2006) 312 arXiv:hep-ph/0512068.

[11] I. Dorsner and P. Fileviez Pérez, "Unification versus proton decay in SU(5)," arXiv:hep-ph/0606062

[12] S. Abdullin and F. Charles, "Study of leptoquark pair production at the LHC with the CMS detector," Phys. Lett. B 464 (1999) 223 arXiv:hep-ph/9905396.

V. A. Mitsou, N. C. Benekos, I. Panagoulias and T. D. Papadopoulou, "Prospects for scalar leptoquark discovery at the LHC," Czech. J. Phys. 55 (2005) B659 arXiv:hep-ph/0411189. 